\title{
Los robots sociales como promotores de la comunicación en los Trastornos del Espectro Autista (TEA)
}

\author{
Robôs sociais como promotores da comunicação em Transtornos do Espectro Autista (TEA)
}

Social robots as promoters of communication in the Autism Spectrum Disorders (ASD)

\author{
Virgina Pinel \\ Laura Aguiló Rendón \\ Daniel Adrover-Roig \\ Universidad de las Islas Baleares, Islas Baleares, España ${ }^{1}$
}

\begin{abstract}
Resumen: La complejidad del Trastorno de Espectro Autista y sus posibles manifestaciones está provocando que, durante las últimas décadas, se esté desarrollando un método de intervención muy prometedor, la terapia asistida con robots. Los desarrollos de la tecnología y los resultados prometedores de los estudios realizados sobre la cuestión, junto con la reciente incorporación de la robótica al contexto educativo hacen que nos planteemos la viabilidad de este método de intervención dentro de un contexto escolar. El presente documento determina los últimos avances del método a partir de las observaciones realizadas por expertos en la cuestión y se analizan las implicaciones positivas que los robots sociales pueden tener para el tratamiento de dicho trastorno. Con todo ello se ofrece una nueva visión a la intervención del autismo en la educación.
\end{abstract}

Palabras clave: Autismo; Robots sociales; Intervención; Educación; Recurso

Resumo: A complexidade do Transtorno do Espectro Autista e sua possível aparência levaram ao desenvolvimento durante a última década de um método de intervenção muito promissor: os robôs assistidos por terapia. O desenvolvimento tecnológico e os resultados promissores dos estudos realizados sobre o assunto, juntamente com a recente incorporação robótica no contexto educacional, fazem com que nós contemplemos a viabilidade de introduzir este método de intervenção no contexto educacional. O presente estudo demarca os últimos avanços deste método a partir da contribuição de especialistas e analisa as conseqüências positivas que os robôs sociais podem ter para o tratamento do transtorno do espectro autista. Nós fornecemos aqui uma nova visão da intervenção do autismo no contexto educacional.

Palavras-chave: Autismo; Robô social; Intervenção; Educação; Recurso

\begin{abstract}
The complexity of the Autism Spectrum Disorder and its possible appearance has lead to the development during the latest decade of a very promising method of intervention: the therapy-assisted robots. The technological development and the promising results of the studies carried out on the matter, along with the recent robotic incorporation in to educational context makes that we contemplate the viability of introducing this intervention method within the educational context. The present study demarcates the latest advances of this method from the contribution of experts and analyses the positive consequences that the social robots can have for the Autism Spectrum Disorder treatment. We provide here a new vision of autism intervention in educational context.
\end{abstract}

Keywords: Autism; Social robot; Intervention; Education; Resource 


\section{Introducción}

El Trastorno de Espectro Autista, en adelante TEA, se caracteriza por una afección en el neurodesarrollo que provoca un persistente deterioro significativo del dominio de la comunicación social unido a patrones restringidos y repetitivos de comportamiento, actividades o intereses. (AMERICAN PSYCHIATRIC ASSOCIATION, 2014). Todo ello hace que las personas que presentan dicho trastorno perciban la realidad de una forma diferente, dificultándoles la conexión con el entorno y provocando que su desarrollo se vea alterado de forma significativa. Las investigaciones sobre el origen de dicha patología no son concluyentes y, aunque todavía no se conoce con certeza la causa, el diagnostico se realiza mediante pruebas físicas, psicológicas y a partir de la identificación de patrones de conducta (WING, 2007)

Según el DSM-V ${ }^{2}$ (American Psychiatric Association, 2014) los criterios diagnósticos de TEA son: deficiencias en la comunicación e interacción social, patrones restrictivos y repetitivos de comportamiento, intereses o actividades. Estos criterios se pueden englobar dentro de lo que Lorna Wing (2007) describe como la triada de deficiencias que engloba las conductas deficientes o alteradas, en individuos con TEA, relativas a la interacción social, la comunicación y la imaginación. La dificultad en cuanto al tratamiento de dicho trastorno reside en los diferentes grados y combinaciones en los que este puede estar presente en los individuos. Psicólogos y expertos han desarrollado planes de tratamiento (biomédicos, terapéuticos y educativos) para mejorar las deficiencias presentes en los niños y adultos con TEA (ALEXANDER et al., 2011).

La prevalencia de los trastornos de espectro autista se ha incrementado en los últimos años llegando a las más de 450.000 personas diagnosticadas en España (MINISTERIO DE SANIDAD, SERVICIOS SOCIALES E IGUALDAD, 2015). Este incremento de los últimos cuarenta años junto con las dificultades de la intervención deriva en la búsqueda de tratamientos alternativos que ofrezcan buenos resultados y permitan disminuir las dificultades del trastorno.

En los últimos años se ha combinado el enfoque psicológico y los avances de ingeniería y la robótica para ofrecer una alternativa en el tratamiento de niños y adultos con TEA, la terapia asistida con robots. Es un método de intervención que, según varias autores y estudios que se detallarán en los próximos apartados, está ofreciendo muy buenos resultados en cuanto al tratamiento de niños con TEA. Según Juan Carlos Cruz et al. (2014) "Los niños con TEA tienen una gran afinidad hacia los juguetes

\footnotetext{
2 Manual de Diagnóstico y Estadístico de los Trastorno Mentales
}

mecánicos, especialmente los robots". A pesar de la escasez de estudios de seguimiento que corroboren la efectividad a largo plazo de dicha terapia, durante las sesiones con robots los sujetos con TEA manifiestan mejoras en las habilidades sociales y comunicativas: mejoran el lenguaje espontáneo, conectan al sujeto con el entorno, provocan comportamientos sociales hacia los robots y reducen los comportamientos repetitivos y estereotipados (PENNISI et al., 2015)

Aunque las aplicaciones que se han realizado hasta ahora de la dicha metodología hayan sido, en su mayoría, en el ámbito terapéutico, también hay algunos estudios realizados en contextos escolares tales como el proyecto AURORA y Robot4Autism que analizaremos en el presente trabajo. Dados los prometedores beneficios que pueden ofrecer este tipo de intervención, el aumento progresivo de casos con TEA y la complejidad de tratamiento del trastorno que nos ocupa es necesario que esta terapia se incluya dentro de un contexto educativo para así ofrecer una mejor respuesta a la necesidad de los alumnos y que esto mejore el tratamiento del mayor número de casos posibles. En la actualidad, el autismo, como ya hemos indicado anteriormente, no tiene cura aunque la intervención temprana y los diferentes tratamientos pueden mejorar la calidad de vida e independencia de este colectivo (ADMONI; MATARIC; SCASSELLATI, 2012). Hay diferentes tratamientos: biomédicos, terapéuticos y educativos (ALEXANDER et al., 2011). El objetivo de todos ellos es mejorar la calidad de vida y de interacción social, al ser esta última la vertiente más afectada. $\mathrm{M}^{\mathrm{a}}$ José Yolanda Hernández et al. (2007) defienden que "El objetivo primordial será el de enseñar de forma explícita lo que no se ha podido aprender de forma natural, y adaptar esa enseñanza a cada individuo en particular".

A nivel terapéutico y educativo, hay multitud de programas de intervención como el Análisis conductual Aplicado, la Intervención Conductual Naturalista, el Modelo evolutivo pragmático, la metodología TEACCH (HERNÁNDEZ et al., 2007) (DAUTENHAHN, 1999) fisioterapia, musicoterapia, aromaterapia, técnicas de relajación, danza (WING, 2007) o terapia asistida con animales sin olvidar la influencia de las nuevas tecnologías y el surgimiento de nuevos mecanismos y sistemas para el mejor tratamiento de niños con TEA.

A todo ello, gracias al rápido progreso de la tecnología y en especial la robótica, se suman nuevas posibilidades en la intervención de TEA (PENNISI et al., 2015). Las diferentes terapias e intervenciones permiten dar respuesta a las necesidades de los niños que padecen este trastorno y la identificación e intervención temprana es sumamente importante (DICLSTEIN-FISCHER; FICHER, 2014). Pues, como bien indican Cabibihan 
et al. (2013) "debido a la naturaleza de la enfermedad y a las grandes variaciones en los síntomas, no se puede establecer un solo enfoque como el mejor ya que el modelo de terapia que puede funcionar bien con un niño puedo no funcionar bien en absoluto con otro".

\subsection{Robots sociales y otros usos}

Los robots sociales, también denominados como "robots interactivos socialmente", "artefactos relacionales" o "juguetes robóticos" se han enmarcado en situaciones sociales de interacción entre un individuo y un robot en contextos de juego, educación y terapéutico. Un prototipo de este tipo de dispositivos es Furby (FERNAEUS et al., 2010).

Por tanto, entendemos robot social como:

robots destinados a actividades básicas de ocio tales como el juego, la creatividad, el aprendizaje, el entretenimiento y la relajación. (...) son juguetes interactivos y tienen un componente de software, el cual los distingue de otros mecanismos o artefactos de baja tecnología (FERNAEUS et al., 2010).

La diferencia básica entre un robot social y cualquier otro juguete, herramienta o instrumento es su interacción con el entorno. A diferencia de otros aparatos, los robots sociales están diseñados para interactuar directamente con el mundo de su alrededor (Breazeal, 2009; Fernaeus et al., 2010;) como bien indican Diclstein-Fischer y Ficher (2014) "están diseñados con la intención de comunicarse e interactuar con los seres humanos".

Según Dautenhahn (2007), los robots sociales o interactivos presentan una serie de características: expresan y/o perciben las emociones, comunican con diálogo de alto nivel o mediante comunicación no verbal, reconocen a otros agentes, establecen o mantienen relaciones sociales, utilizan señales naturales (mirada, gestos, etc.), tiene una personalidad o carácter distintivo y pueden aprender o desarrollar competencias sociales. La presencia de estas características en mayor o menor grado son diferentes en cada robot.

Juan Carlos Cruz y Yeliza Andrea Salazar (2014) apuntan a que una de las grandes ventajas de estos dispositivos es que pueden programarse para responder a las diferentes situaciones y también pueden aprender y cambiar la forma de responder al mundo. Por lo tanto, sus respuestas e interacciones se vuelven más sofisticadas y eso permite que la motivación y atención del niño vaya in crescendo. Sin embargo, se han realizado pocos estudios sobre el efecto a largo plazo de los beneficios de este tipo de terapias y algunos autores apuntan que podría conducir a una falta de apego humano (TANAKA; KIMURA, 2009).
El uso de robots en terapia es limitado por el gran coste que supone y la falta de disponibilidad de estos dispositivos que normalmente se limitan al entorno clínico o privado (ALEXANDER et al., 2011). No obstante, recientemente se están comercializando algunos de estos robots a un coste medianamente asequible y esto permitiría expandir este tipo de terapias a otros ámbitos como puede ser el educativo.

Estos robots surgen de una tendencia robótica reciente llamada robótica de asistencia social, una subárea de la robótica que pretende diseñar robots para ayudar y cubrir las necesidades especiales de personas con dificultades sociales, físicas o de interacción (BERNIER et al., 2012).

En la actualidad se está investigando el uso de tales robots como una herramienta para la práctica de habilidades sociales, la formación empática, la enseñanza de idiomas o en terapias conductuales (AHLUWALIA et al., 2012). De cada vez más se utilizan en rehabilitación, terapia y educación (CASTIELLO et al., 2008). También se han investigado como herramienta de apoyo para personas de edad avanzada o con déficit físicos como el derrame cerebral y parálisis parcial de las extremidades (BERNIER et al., 2012). Además. Colton et al. (2008) indican que del uso de robots sociales también se podrían beneficiar otro colectivo de la población de niños como los niños con trastorno específico del lenguaje (TEL). En este sentido, como indicaremos en próximos apartados, este tipo de terapias permite la aparición de interacciones triádicas entre el niño, robot y terapeuta que podría desencadenar en interacciones sociales entre el niño y el terapeuta (COLTON et al., 2008). Muchos de los usos se han centrado en personas con trastornos de la comunicación. Gideki Kozima, Cocoro Nakagawa y Yasuda (2005) describen el uso de robots sociales para la interacción en actividades terapéuticas en niños con autismo, trastorno generalizado del desarrollo y otros trastornos del desarrollo de la comunicación y concluyen que, para estas personas, esta tecnología ayudará a mejorar su calidad de vida social. Otros autores como Cabibihan et al. (2013) subrayan que es una herramienta muy utilizada para enseñar habilidades a los niños con autismo, jugar con ellos y provocar conductas deseadas.

Como veremos más adelante, el uso de robots en terapias o intervención, especialmente para el tratamiento del TEA puede mejorar la capacidad y eficacia de las intervenciones (DICLSTEIN-FISCHER; FICHER, 2014).

\section{Terapia asistida con robots sociales}

Como hemos visto anteriormente, uno de los usos más generalizados de los robots sociales que se 
está estudiado actualmente es la terapia en niños con TEA. Según Paola Pennisi et al. (2015) los niños con autismo muestran una clara atracción hacia los sistemas tecnológicos. En relación a esto, autores como Juan Carlos Cruz y Yeliza Andrea Salazar (2014) añaden que "los niños autistas tienen una gran afinidad hacia los juguetes mecánicos, especialmente los robots. La previsibilidad de comportamiento repetitivo y monótono del robot es el factor reconfortante que hace que los niños autistas tengan una gran atracción por robots". Por ello, y por otras razones que se analizarán en próximos apartados, se cree que se trata de un método viable para tratar este tipo de trastorno.

Tal y como indican Paola Pennisi et al. (2015) " $L a$ robótica social podría ser un método prometedor para el tratamiento de los trastornos del espectro autista (TEA)". Esta afirmación la corroboran Gideki Kozima, Cocoro Nakagawa y Yasuda (2005) en su estudio realizado con Keepon, robot al que haremos referencia más adelante, en el que aseguran que los niños con TEA mostraron expresiones faciales que ni individuos del entorno más próximo al niño habían visto antes e incluso desarrollaron conductas pro-sociales hacia el robot.

Las plataformas robóticas son un método particularmente interesante para interactuar con los niños con autismo, porque propicia en ellos un abandono de su mundo introspectivo y lo invita a responder a los estímulos producidos por el robot (CRUZ; SALAZAR, 2014). Algunos factores que hacen que los robots sociales sean un medio ideal para la terapia del TEA son: su simplicidad, su adaptabilidad de comportamiento a los diferentes escenarios y su posibilidad de ofrecer una interacción esperada y más simple (AKHTAR et al., 2012). Además Ueyama (2015) sostiene que estos robots animan a los niños con TEA a tomar la iniciativa y estimular las respuestas emocionales.

En definitiva, el uso de estos robots es utilizado para ayudar a los niños con TEA a comunicarse, interactuar, reconocer emociones y desarrollar su competencia social (UEYAMA, 2015) mediante el entrenamiento de la mirada compartida y la atención conjunta, la mejora de la imitación y la toma de turnos, la enseñanza de emociones faciales y corporales y la iniciación de interacciones sociales (BARAKOVA, 2011).

La mayoría de los estudios realizados hasta el momento se han llevado a cabo en laboratorios o entornos institucionales como parte de una educación específica o programas terapéuticos (FERNAEUS et al., 2010). Se han demostrado los beneficios de la robótica para la interacción social en estudios de casos de tres o cuatro niños pero hay pocos estudios a gran escala (DIEHL et al., 2012 citado por BERNIER et al., 2013).
Pennisi et al. (2015) realizaron una revisión de los diferentes estudios sobre la cuestión llevados a cabo hasta el momento. En cuestiones de rendimiento de los niños con TEA en condiciones humanas o con el robot los autores reflejan que hasta el año 2015, 13 estudios señalan mejores actuaciones hacia el robot que hacia un agente humano. Además, durante el juego el contacto visual, táctil, la manipulación, la postura y la producción verbal eran mejores en las sesiones con robot. Según los mismos autores se han realizado dieciséis estudios que analizaban el comportamiento social: ocho de estos mostraron que el robot puede ser un mejor estímulo que un humano para la mejora de los comportamientos sociales, catorce indican que los niños con TEA muestran comportamientos sociales hacia el robot y nueve determinaron mejores resultados cuando el robot ejercía como mediador. Los mismos autores indican que tres de los cuatro estudios que analizan la mejora del lenguaje con este tipo de terapia corroboran que el robot promueve la mejora del lenguaje. Los autores apuntan que, en cuanto a la imitación en este tipo de terapia, cuatro mostraron que los niños con TEA mejorar la imitación si se utilizan robots. De hecho, en uno de los estudios desarrollado con 24 participantes con TEA se demuestra la mejora de las habilidades del lenguaje en la interacción triádica (KIM et al., 2013; citado por PENNISI et al., 2015).

Todos los estudios que analizan Pennisi et al. (2015) muestran que los robots pueden ser buenos motivadores y ayudar a atraer la atención de los niños a la tarea (LEE; TAKEHASHI; NAGAI; OBINATA et al., 2012; LEE; OBINATA, 2013; WAINER et al., 2010; WAINER; DAUTENHAHN et al., 2014.; WAINER; ROBINS et al., 2014.; YEE et al., 2012.; YIN et al., 2013).

A raíz de los diferentes estudios realización se han desarrollado diferentes proyectos que utilizan la robótica. Uno de ellos es el Proyecto Robota que se centra en la investigación del desarrollo de juguetes robóticos educativos e investiga la posibilidad de utilizar el robot para evaluar la capacidad de imitación de los niños y enseñarles comportamientos simples (COSTA et al., 2010). Otro de los proyectos que se centra en el posible uso de los robots para ayudar a personas con problemas de comunicación a adquirir y/o mantener sus habilidades y capacidades comunicativas es el Proyecto ComunicationCare (KOZIMA; NAKAGAWA; YASUDA, 2005). Otro de los proyectos en funcionamiento es LEGO terapia que utiliza la herramienta LEGO Mindstrom que según Cruz y Salazar (2014) es muy eficaz para trabajar con niños con TEA. Además, los mismos autores describen otros proyectos en desarrollo como IROMEC, KEEPOM o layROB además del Proyecto AURORA y Robot4Autism que analizaremos más adelante dada su vinculación con el entorno educativo. 


\subsection{Ventajas y desventajas de la terapia con robots sociales}

\subsubsection{Ventajas del uso de robots sociales}

Las implicaciones positivas del uso de estos dispositivos para la terapia del autismo son muy numerosas (PENNISI et al., 2015; CABIBIHAN et al., 2013). Una de las grandes ventajas que ofrece la terapia asistida con robots es que permite a los individuos con TEA conectar con el entorno de una manera más fácil (PENNISI et al., 2015; DAUTENHAHN, 1999). Laurie DicksteinFischer y Gregory S. Ficher (2014) añaden que éstos pueden aumentar las posibilidades de interacción social con otros agentes humanos. Además, a diferencia de otros programas y aplicaciones virtuales, el robot permite una interacción multimodal de forma natural mediante gestos, expresiones, contacto, etc. lo que hace que resulten aún más atractivos (CABIBIHAN et al., 2013). Cristina A. Costescu, Daniel O. David y Bram Vanderbroght (2015) sostienen que los niños con TEA parecen disfrutar más de la tarea interactuando con el robot en comparación con un adulto. Además, Ueyama (2015) defiende que el uso de robots mejora la participación de los niños con TEA durante la terapia y que la interacción con el robot no les resulta incómoda dado que se ha observado que la respuesta emocional a estos dispositivos mejora en relación a la interacción con humanos. Además, Paola Pennisi et al. (2015) indican que algunas implicaciones positivas del uso de estos dispositivos son que los niños con autismo reaccionan mejor a un robot que a un agente humano y reducen los comportamientos estereotipados y repetitivos. Asimismo, los robots provocaban una gran cantidad de comportamientos sociales por parte de los niños con autismo y mejoraban el lenguaje espontáneo de los niños con TEA durante las sesiones.

Como ya hemos avanzado, el autismo se caracteriza por una tríada de deficiencias (WING, 2007) junto a comportamientos estereotipados y dificultades en la imitación y, este tipo de terapia, contribuiría a mejorar cada una de las vertientes afectadas por dicho trastorno. Para observar con mayor amplitud los beneficios, posibilidades y limitaciones de este tipo de terapia analizaremos los beneficios desde cada una de estas áreas:

\section{Interacción social}

Los robots sociales pueden ser buenos factores desencadenantes de algunas habilidades sociales (PECA et al., 2016) siendo los beneficios del uso de robots sociales en este aspecto:

- Se incita a la generación de una respuesta emocional e imitan y mantienen el contacto visual con mayor frecuencia con robots (ALEXANDER et al., 2011).
- Los niños reconocen mejor las expresiones faciales con los robots que con los seres humanos $\mathrm{y}$, dada la motivación intrínseca, permite avanzar más en la teoría de la mente y facilitar la comprensión de señales sociales complejas (NEILON; ROLLINS, s.f.)

- Eliminan la sobrecarga sensorial y facilita el reconocimiento de las emociones puesto que están programados para mostrar un conjunto básico de emociones (CABIBIHAN et al., 2013)

- Durante la interacción niño-robot al niño le resulta más fácil seguir la mirada del robot hacia un objeto específico y, progresivamente, el niño consigue iniciar el contacto y guiar la intención del robot llegando, por último, a realizar esta misma acción con el terapeuta (CABIBIHAN et al., 2013).

- Ayuda a romper el aislamiento y facilitar la interacción con otras personas (ALEXANDER et al., 2011).

- Pueden ser utilizados no solo como compañeros de juego o mediadores sino también como juguetes que pueden ser programados y adaptados de acuerdo a sus necesidades asegurando que los niños puedan sentirse a gusto en la interacción (CABIBIHAN et al., 2013).

\section{Comunicación}

Los robots tienen un enorme potencial para ser utilizados como un sistema de andamiaje de las habilidades comunicativas de los niños con TEA (AKHTAR et al., 2012). Pues, tal y como indican Pennisi et al. (2015) " $L o s$ datos de la robótica social y de mejora del lenguaje en la terapia del autismo son los más claros". Algunos de los beneficios son:

- Los robots desencadenan respuestas sociales en niños con TEA dado que las habilidades sociales de los robots son muy simples (COLTON et al., 2008).

- El niño ve recompensado su esfuerzo y se anima a iniciar interacciones, no solo con el robot si no también con el terapeuta (CABIBIHAN et al., 2013).

- Es más fácil que los niños sigan instrucciones y no se dejen intimidar por las complejidades de la comunicación verbal y no verbal, el proceso de comunicación es más fácil (CABIBIHAN et al., 2013).

- Los robots pueden ser útiles para enseñarles a esperar una respuesta del interlocutor o a esperar el turno durante un juego (CABIBIHAN et al., 2013).

En definitiva, los robots tienen un enorme potencial para ser utilizados como un sistema de andamiaje de 
las habilidades comunicativas de los niños con TEA (AKHTAR et al., 2012)

\section{Imaginación e Imitación}

Jhon-Jhon Cabibihan et al. (2013) sostienen que la imitación es una de las habilidades que utilizan la mayor parte de robots durante la intervención dado su gran importancia en el proceso de aprendizaje. Muchos estudios han utilizado la imitación espontánea como base de la interacción (DIEHL et al., 2012; RICKS; COLTON, 2010; SCASSELLATI, 2007; KOZIMA et al., 2007; FERRARI et al.; 2009, citado por COSTESCU; DAVID; VANDERBORGHT, 2015). Sin embargo, algunos estudios apuntan que la imitación automática es la normal (BIRD et al., 2007; PIERNO et al., 2008) e incluso el robot podría tener una influencia negativa en la imitación de las palabras (KIM et al., 2013). No obstante, tal y como indican Pennisi et al. (2015) aún se debe estudiar con mayor profundidad la capacidad de los robots de provocar la imitación de expresiones faciales.

\section{Comportamientos estereotipados}

Según Pennisi et al. (2015) los cuatro estudios que analizan este parámetro dan resultados positivos y por tanto, la tasa de comportamientos estereotipados disminuye cuando hay interacción con el robot (SHAMSUDDIN et al., 2013). Sandra Costa et al. (2010) afirma que "Los robots parecen actuar como una herramienta clave para poder llamar la atención de los niños autistas y promover el desarrollo cognitivo $y$ social". Esta motivación inducida por el robot es muy beneficiosa dado que varios estudios apuntan que los niños con TEA se comprometen mejor en la tarea si la información se presenta de una forma atractiva, están más atentos, motivados, tienen mejor rendimiento y disfrutan más de la tarea (COSTESCU; DAVID; VANDERBURGHT, 2015).

\subsection{Desventajas del uso de robots sociales}

Como cualquier otra herramienta, los robots sociales también tienen desventajas, indicadas por algunos autores y se muestran a continuación:

- Los estudios realizados hasta el momento son muy limitados y es necesario aclarar si los beneficios solo se producen durante las sesiones de terapia o son generalizables a otros contextos (PENNISI et al., 2015).

- A pesar de que durante el trascurso de las sesiones se producen mejoras sociales, esto no predice necesariamente los efectos a largo plazo (BERNIER et al., 2013).
- Los robots podrían reforzar la tendencia a caer en un comportamiento estereotipado repetitivo (KLJAJEVIC, 2010).

- Cuando las tareas consisten en que el robot invite al niño a mirar un objeto, el robot puede ser un distractor ya que canaliza la atención del niño (PENNISI et al., 2015).

- La terapia puede ser costosa aunque existen robots en el mercado a un coste moderado disponibles para médicos, padres o maestros (ADMONI; MATARIC; SCASSELLATI, 2012).

- Existen dificultades de organización y el miedo a la complejidad técnica del control del robot hacen generan desconfianza a la hora de implementar este tipo de terapia (BARAKOVA, 2011).

\section{Consideraciones sobre los robots i sobre la terapia}

\subsection{Consideraciones sobre los robots}

Pennisi et al. (2015) argumentan que los robots utilizados como mediadores deberían ser humanoides y tener una cara como por ejemplo FACE, Flobi, Nao, Parlo, Zena, Kaspar o Robota. Además, los robots menos realistas, como los robots de tipo animal, podrían ayudar a centrar la atención en señales sociales necesarias limitando los estímulos distractores y que para trabajar el contacto visual lo deseable es que el robot tenga ojos claramente distinguibles con algún tipo de animación (Ifbot, Pleo, Kaspar, FACE, Flobi, Nao o Zeno).

Jenry Admoni, Maja Mataric y Brian Scassellati (2012) indican que durante la terapia los robots pueden ser presentados como juguetes, ser subordinados que necesitan ayuda del niño durante el juego, ser como padres que estimulan la imitación a través de juegos, tomar la iniciativa en la orientación de las interacciones sociales o bien ser una herramienta utilizada por el terapeuta para guiar al niño y animarlo a imitar su comportamiento e interactuar con él.

Los robots destinados a este tipo de aplicaciones deben ser tan simples como sean posible dado que si estos tienen un comportamiento complejo podrían transmitir demasiada información y podrían asustar y abrumar a los niños con TEA (KOZIMA; NAKAGAWA; YASUDA, 2005). De acuerdo con Cabibihan et al. (2013) los robots destinados para el tratamiento de los niños con TEA deben contar con una serie de requisitos de diseño en relación a la apariencia, funcionalidad, requisitos de seguridad, autonomía, modularidad y adaptabilidad.

- Apariencia: Deben ser visualmente atractivos para captar la atención del niño y deben tener un tamaño que le permita una fácil interacción. Las expresiones faciales deben ser simples y se deben 
evitar rasgos triviales para mejorar la simplicidad. Además el tamaño de los ojos depende del niño dado que algunos se sienten amenazados y los niños tienden a mostrar mayor afinidad hacia los robots no humanoides a pesar de que en algunos casos los robots humanoides permiten una mejor generalización.

- Funcionalidad: deben ofrecer una retroalimentación positiva a través de la respuesta a los estímulos que percibe del entorno. Los robots móviles provocan más comportamientos positivos por parte de estos niños y el robot debe permitir al niño tomar decisiones durante la interacción mediante algún botón manual.

- Requisitos de seguridad: deben ser robustos y no tener bordes afilados.

- Autonomía: deben permitir, por un lado, el control de una secuencia de movimiento por parte del terapeuta, y por otro, mostrar un nivel de autonomía de tal forma que si no es controlado no deje de interactuar con el entorno. No es aconsejable que la autonomía sea completa ya que, tal y como indican los autores, "el terapeuta debe ser capaz de decidir el comportamiento del robot en respuesta al comportamiento del niño. $L a$ presencia de un ser humano en el bucle es vital".

- Modularidad y adaptabilidad: Es deseable que el robot vaya incrementando la complejidad de sus comportamientos y que se adapte al entorno, intereses y preferencia del niño.

El robot debe ser capaz de permitir la imitación y debe tener la capacidad de apuntar a un objeto o persona y dirigir la mirada hacia el mismo punto (COLTON et al., 2008).

Como en la utilización de cualquier otra técnica, la edad mental y la edad cronológica son importantes para que la terapia sea efectiva (CABIBIHAN et al., 2013). Según Juan Carlos Cruz y Yeliza Andrea Salazar (2014), durante la terapia se deben respetar los procedimientos que tienen definidos los terapeutas para interactuar con los niños. Los logros que se consigan durante las terapias deben generalizarse a los entornos del niño, por ello, es importante provocar, durante las intervenciones, interacciones triádicas entre el niño, el robot y otros compañeros para ayudarle a mejorar esta interacción social no solo con el robot, sino también con sus compañeros (CABIBIHAN et al., 2013). Mark B. Colton et al. (2008) también defienden la construcción de esta tríada de relaciones para mejorar la generalización. Estos mismos autores sugieren utilizar el robot como una herramienta que potencia la atención conjunta y que facilite la interacción entre el niño y su cuidador.
Uno de los objetivos de la terapia es permitir una mejor comprensión de la tarea por parte del niño para aumentar si participación y rendimiento (COSTESCU; DAVID; VANDERBORGHT, 2015). Tenemos que tener presente que, tal y como indican Cabibihan et al. (2013), el propósito de las intervenciones es permitir a los niños superar sus deficiencias y ayudarles a comprender mejor el mundo.

Un aspecto importante durante la terapia es que el robot debe ser introducido como un mediador entre el niño y las persona co-presentes (COLTON et al., 2008; COSTA, s.f.). En un estudio realizado se mostró como el niño con TEA tuvo mayor contacto visual, actitud más amplia y habló más con sus compañeros de juego (WAINER; ROBINS et al., 2014 citado por Pennisi et al., 2015). Además, según Dautenhahn (1999) esto puede ayudar a que el niño se acostumbre a los estilos básicos de interacción típicos entre los seres humanos, por tanto, el robot debe introducirse como un agente que interactúa y va ampliando sus estímulos gradualmente para responder a los comportamientos del niño (DAUTENHAHN, 1999). Según Costa et al. (2010) el robot puede ser utilizado como un complemento para la terapia diaria o como un sustituto del terapeuta. Aunque, no todos los autores están de acuerdo con esto, como por ejemplo, Costescu, David y Vanderborght (2015) que sostienen que el robot debe ser visto como una herramienta tecnológica.

Según Barakova (2011) los terapeutas son un elemento clave en el proceso por dos motivos: sólo su conocimiento puede hacer que los programas con robots sean eficaces y sin la participación de éstos no se asegura la adquisición de habilidades con la ayuda del robot. Por ello, durante la terapia la función del robot no es reemplazar los roles del terapeuta sino más bien actuar como agente de interacción para mantener el compromiso de los niños autistas durante la terapia (AKHTAR et al., 2012). Pues, tal y como indican Colton et al. (2008) el terapeuta tiene una gran importancia en el logro de beneficios.

Debemos recordar que el robot es una herramienta y por el simple hecho de utilizarla no se asegura el logro de los objetivos; en consecuencia, Cabibihan et al. (2013) recuerdan que las sesiones deben estar constituidas por actividades que nos permitan lograr los objetivos planteados y provocar los comportamientos deseados. Algunos de los aspectos que siguieren trabajar dichos autores son: la imitación, el contacto visual, la atención conjunta, la toma de turnos, el reconocimiento y expresión de emociones, la iniciativa en la interacción y las interacciones tríadicas. Pues, tal y como indican Neilon y Rollins (s.f.) "la terapia no puede estar constituida únicamente por sesiones con un robot" y una vez que ha mostrado el dominio de las habilidades 
durante la interacción con el robot, estas se deben generalizar mediante actividades estructuradas a la vida real.

\section{Robots sociales en contexto educativo}

Fumihide Tanaka y Takeshi Kimura (2009) realizaron un estudio en un aula de educación infantil de California utilizando el robot RUBI. Del estudio concluyeron que el robot era una muy buena herramienta para obtener y mantener la atención de los niños. Además apunta que los robots destinados a la educación deben ayudar y apoyar a los docentes bajo su control, en definitiva, "es una herramienta de los docentes para enriquecer el entorno educativo". Se han llevado a cabo algunos estudios cuantitativos en escuelas con pequeños humanoides como QRIO y Robivie (TANAKA; KIMURA, 2009).

Como hemos avanzado en anteriores apartados, dos de los proyectos que se realizan actualmente se llevan a cabo en entornos educativos. Nos referimos al proyecto AURORA y al proyecto Robot4Autism, que explicaremos a continuación. El Proyecto AURORA es un proyecto realizado en la escuela de primaria Bentfield de Reino Unido (BILLARD et al., 2004). Tal y como indican Cruz y Salazar (2014) el proyecto investiga la función terapéutica y educativa que pueden cumplir los robots para los niños con TEA y utiliza dos robots: Robota y Kaspar. Con el proyecto se pretende enseñar habilidades sociales básicas a los niños con TEA fomentando el aspecto didáctico y las relaciones tríadicas. Los objetivos del proyecto son Ayudar a los niños con TEA a vincularse con el mundo social y estudiar la interacción humanorobot (DUTENHAHN, 1999).

Robot4Autism se centra en niños con TEA en edad escolar y busca trabajar las habilidades sociales relevantes a partir de robots humanoides y tabletas (NEILON; ROLLINS, s.f.). Fumihide Tanaka y Takeshi Kimura (2010) están convencidos del potencial positivo de la tecnología robótica como herramienta de apoyo para los maestros para la mejora de la calidad de la educación infantil. Kerstin Dautenhahn (1999) ya anunciaba que las máquinas inteligentes humanoides no formaban parte de nuestras vidas y que sería un proceso largo y difícil. "El equilibrio entre los beneficios y los riesgos de esta tecnología es siempre dinámico" (TANAKA; KIMURA, 2010).

\section{Referencias}

ADMONI, H.; MATARIC, M.; SCASSELLATI, B. Robots for Use in Autism Research. Annual Review of Biomedical Engineering, v. 14, p. 275-294, 2012. doi: 10.1146/annurevbioeng-071811-150036
AHLUWALIA, A. et al. Realistic Humanlike Robots for Treatment of ASD, Social Training, and Research; Shown to Appeal to Youths with ASD, Cause Physiological Arousal, and Increase Humanto-Human Social Engagement. Association for Computing Machinery. The 5th ACM International Conference on PErvasive Technologies Related to Assistive Environments, 2012.

AKHTAR, F. et al. Humanoid Robot NAO Interacting with Autistic Children of Moderately Impaired Intelligence to Augment Communication Skills. International Symposium on Robotics and Intelligent Sensors 2012, p. 1533-1538, 2012. doi: 10.1016/j.proeng.2012.07.346

ALEXANDER, E. el al. An Affordable Compact Humanoid Robot for autism Spectrum Disorder Interventions in Children. Engineering in Medicine and Biology Society (Ed.). 33rd Annual International conference of the IEEE EMBS, p. 53195322, 2011. doi: 10.1109/IEMBS.2011.6091316

ASOCIACIÓN Estadounidense de Psiquiatría. Manual de diagnóstico y estadístico de los trastornos mentales (DSM-5). 5. ed. Madrid: Panamericana, 2014.

BARAKOVA, E. Robots for social training of autistic children. Empowering the therapists in intensive training programs. IEEE (Ed.), Information and Communication Technologies (WICT), 2011 World Congress on. 2011. p. 14-19. doi: 10.1109/ WICT.2011.6141197

BERNIER, E. et al. Social Robots as Embedded Reinforcers of Social Behavior in Children with Autism. Journal Autism Dev Disord, v. 43, p. 1038-1049, 2012. doi: 10.1007/s10803012-1645-2

BILLARD, A. et al. Effects of repeated Exposure to a Humanoid Robot on Children with Autism. In: KEATES, S. et al. (Ed.). Designing a More Inclusive Word, 2004. p. 225-236. doi: 10.1007/978-0-85729-372-5 23

BREAZEAL, C. Role of expressive behaviour for robots that learn from people. Psychological Transactions of The Royal Society, v. 364, p. 3527-3538, 2009. doi: 10.1098/rstb.2009.0157

CABIBIHAN, J. et al. Why robots? A survey on the roles and benefits of social robots in the therapy of children with autism. International Journal of Social Robotics, v. 5, n. 4, p. 593-618, 2013. doi: 10.1007/s12369-013-0202-2

CASTIELLO, U. et al. Robotic movement elicits visuomotor priming in children with autism. Neuropsychologia, v. 46, p. 448-454, 2008. doi: 10.1016/j.neuropsychologia.2007.08.020

COLTON, M.; RICKS, D. Trends and Considerations in RobotAssisted Autism Therapy. IEEE (Ed.). IEEE, Conference: Robotics and Automation (ICRA), 2008. doi: 10.1109/ ROBOT.2010.5509327

COLTON, M. et al. Toward Therapist-in-the-Loop Assistive Robotics for Children with Autism and Specific Language Impairment. AISB New Frontiers in Human-Robot Interaction Symposium Conference, 2008. doi: 10.1.1.160.125

COSTA, S. Robots as Tools to Help Children with ASD to Identify Emotions.

COSTA, S. et al. Promoting interaction amongst autistic adolescents using robots. IEEE (Ed.). Engineering in Medicine and Biology Society (EMBC), 2010 Annual International Conference of the IEEE, 2010. p. 3856-3859. doi: 10.1109/ IEMBS.2010.5627905 
COSTESCU, C.; DAVID, D.; VANDERBORGHT, B. Reversal Learning Task in Children with Autism Spectrum Disorder: A Robot-Based Approach. Journal Autism Dev Disord, v. 45, p. 3715-3725, 2015. doi: 10.1007/s10803-014-2319-Z

CRUZ, J. C.; SALAZAR, Y. A. Aplicación robótica para realizar terapias en niños con autismo, 2014.

DAUTENHAN, K. Robots as social actors: Aurora and the case of autism. Department of Cybernetics, University of Reading; MIT Artificial Intelligence Laboratory (Ed.). In: Proceedings of the Third Cognitive Technology Conference, 1999. Doi: 10.1.1.190.1767

DAUTENHAN, K. Social intelligent robots: dimensions of human-robot interaction. Psicological Transactions of The Royal Society, v. 362, p. 679-704, 2007. doi: 10.1098/ rstb.2006.2004

DICKSTEIN-FISCHER, L.; FISCHER, G. S. Combining Psychological and Engineering Approaches to Utilizing Social Robots with Children with Autism. Engineering in Medicine and Biology Society (Ed.). 36th Annual International conference of the IEEE, 2014. p. 792-795. doi: 10.1109/ EMBC.2014.6943710

FERNAEUS, Y. et al. How do you Play with a Robotic Toy Animal? A long-term study of Pleo. 9th International Conference on Interaction Design and Children. 2010. p. 39-48. doi: 10.1145/1810543.1810549

KLJAJEVIC, V. Syntactic deficits in autism: Can interactive technologies help? Curr Top Neurol Psychiatr Relat Discip., v. 18 , n. 2 , p. $38-45,2010$.

KOZIMA, H.; NAKAGAWA, C.; YASUDA, Y. Interactive Robots for communication-Care: A Case-Study in Autims Therapy. IEEE (Ed.). IEEE International Workshop on Robots and Human Interactive Communication. 2005. p. 341-346. doi: 10.1109/ROMAN.2005.1513802
MINISTERIO de Sanidad, Servicios Sociales e Igualdad. Estrategia Española en Trastorno del Espectro del Autismo, 2015.

NEILON, M.; ROLLINS, P. Technology-aided Instruction is now classified as one of the 27 intervention practices for children with autism.

PECA, A. et al. Children with Autism Spectrum Disorders Make a Fruit Salad with Probo, the Social Robot: An Interaction Study. Journal Autism Dev Disord, v. 46, p. 113-126, 2016. doi: 10.1007/s10803-015-2556-9

PENNISI, P. et al. Autism and social robotics: A systematic review. Autism Research, v. 9, n. 2, p. 165-183, 2015. doi: 10.1002/aur.1527

TANAKA, F.; KIMURA, T. The Use of Robots in Early Education: A Scenario Based on Ethical Consideration. IEEE (Ed.). The 18th IEEE International Symposium on Robot and Human Interactive Communication. 2009. p. 558-560. doi: 10.1109/ROMAN.2009.5326227

TANAKA, F.; KIMURA, T. Care-receiving Robot as a Tool of Teachers in Child Education. Interaction Studies, v. 11, n. 2, p. 263-268, 2010. doi: 10.1075/is.11.2.14tan

UEYAMA, Y. A Bayesian Model of the Uncanny Valley Effect for Explaining the Effects of Therapeutic Robots in Autism Spectrum Disorder. PLoS ONE, v. 10, n. 9, 2015. doi: 10.1371/ journal.pone.0138642

WING, L. Historia: Evolución de las ideas sobre los trastornos del espectro autista. El autismo en niños y adultos: Una guía para la familia. Barcelona: Paidos, 1998.

Recebido: 30/09/2017

Aprovado: 07/01/2018

Contato:

Daniel Adrover Roig <danieladrover@gmail.com> 